Ann. Zootech., I974, 23 (2), I93-2I2.

\title{
ÉTUDE DU COMPORTEMENT ALIMENTAIRE ET MÉRYCIQUE DE MOUTONS RECEVANT DES FOURRAGES VERTS HACHÉS
}

\author{
J.-P. DULPHY et C. DEMARQUILLY \\ avec la collaboration technique de J. M. Borsseau, \\ H. Bousquet et L. Lhotelier \\ Laboratoire des Aliments, \\ Centre de Recherches de Clermont-Ferrand, I. N.R. A., \\ Theix, 63110 Beaumont
}

\section{RÉSUMÉ}

Dans le cadre des études faites sur l'ingestibilité et la digestibilité des fourrages verts, nous avons enregistré les manifestations du comportement alimentaire et merycique de moutons à l'auge afin de définir quelques facteurs de variations de ce comportement. Ces observations ont porté sur 174 échantillons de fourrages verts appartenant à 7 espèces végétales (tabl. I).

Les principaux résultats qui ressortent de ces études sont les suivants :

- La durée journalière d'ingestion des fourrages verts est comprise entre 250 et $400 \mathrm{mn}$. Cette durée diminue principalement lorsque les fourrages vieillissent, c'est-à-dire lorsque leur ingestibilité et leur digestibilité diminuent (tabl. 2 et 4 ).

- Le nombre de repas effectués par jour est très variable (4 à I6). Il varie d'une espèce végétale à l'autre et surtout il diminue lorsque la plante vicillit. Cette diminution est liée à celle des quantités ingérées de matière sèche (tabl. 2 et 5 ).

- La durée unitaire d'ingestion (3 à $7 \mathrm{mn} / \mathrm{g} / \mathrm{kg} \quad \mathrm{P}^{0,75}$ ) augmente très lentement avec l'âge des fourrages. Elle est plus élevée pour les graminées que pour les légumineuses (tabl. 2 et 4 ).

- La durée journalière de rumination (300 à 650 minutes) augmente avec l'âge des fourrages et surtout avec leur teneur en cellulose brute (tabl. 6 et 7). Cette durée semble se stabiliser autour d'une valeur comprise entre 525 et 575 minutes par jour suivant l'espèce végétale ou l'animal dès que la teneur en cellulose brute de la plante atteint une valeur voisine de 30 p. Ioo (fig. I).

- La durée unitaire de rumination (4 à $12 \mathrm{mn} / \mathrm{g} / \mathrm{kg} \mathrm{P}^{\mathrm{P}, 75}$ ) augmente relativement rapidement lorsque la digestibilité et l'ingestibilité du fourrage baissent (tabl. 6 et 7).

- La durée journalière de mastication (600 à 950 minutes) est par contre relativement constante pour une espèce végétale donnée au cours de son vieillissement. Cela explique pourquoi la durée unitaire de mastication est reliée étroitement et inversement à la quantité de matière sèche ingérée. Cette durée unitaire peut constituer "an index of fibrousness characteristic of roughages" comme le propose BaLCH (I97I) (tabl. 6 et 8).

Ces résultats sont discutés et nous avons essayé de les relier aux facteurs connus conditionnant la régulation physique de l'appétit chez le ruminant, en particulier aux vitesses de digestion dans le rumen et de vidange de ce rumen (schéma I). Nous nous sommes demandés aussi si la durée de rumination journalière ne pouvait pas devenir un facteur limitant les quantités ingérées de fourrages lorsque leur teneur en cellulose brute atteignait $3^{\circ} \mathrm{p}$. I oo de la matière sèche. 


\section{INTRODUCTION}

Certains auteurs, tels que GoRdon (I965), W ELLCH et SMITH (Ig6ga et $b$ ), KERBAA (I969), ont montré qu'il existe des liaisons directes entre les durées d'ingestion et de rumination et les quantités de fourrages ingérées par les ruminants. Quant à nous, dans un article récent (DULPHY, I972), nous avons indiqué que l'examen des manifestations du comportement alimentaire et mérycique des moutons pouvait être une voie intéressante pour l'étude de la régulation de l'ingestion des animaux recevant des fourrages conservés. De plus, pour nos études sur 1'ingestibilité des ensilages, il nous faut connaître les modifications du comportement entraînées par le mode de conservation. Il était donc intéressant de s'intéresser tout d'abord aux manifestations du comportement des animaux recevant uniquement des fourrages verts. C'est pourquoi nous avons réuni les résultats enregistrés depuis ig66 dans le cadre des études faites sur la digestibilité et l'ingestibilité des fourrages verts et conservés (DEMARQUILLY et WEISS, 1970) et nous avons regroupé ceux relatifs aux plantes prairiales afin d'étudier quelques facteurs de variation du comportement des moutons alimentés avec ces plantes à l'auge.

\section{MATÉRIEI，ET MÉTHODES}

De 1966 à 197 I, les manifestations du comportement alimentaire des moutons ont été enregistrées pour I 74 échantillons de fourrages verts dont on mesurait I'évolution de la valeur alimentaire (digestibilité et ingestibilité) avec le stade de végétation au cours du I er cycle (pendant 6 à 8 semaines) ou avec l'âge pour les repousses (de la $4^{\mathrm{e}}$ à la $8^{\mathrm{e}}$ ou $9^{\mathrm{e}}$ semaine environ). Le nombre des espèces et des cycles étudiés est reporté au tableau I avec les caractéristiques moyennes des fourrages.

Les manifestations du comportement alimentaire et mérycique ont été enregistrées selon la technique de Ruckebusch (I963) pendant cinq jours consécutifs (du lundi matin à 8 heures au samedi matin à 8 heures) sur 2 (I966), 3 (I967 à I970) ou 4 (I97I) des 4 moutons en cage à métabolisme utilisés pour mesurer l'évolution de la valeur alimentaire des fourrages verts avec l'âge au cours des cycles successifs de végétation. De ig66 à 1970, les mesures ont été faites une semaine sur deux alors qu'elles ont été effectuées chaque semaine en r97I.

Les moutons utilisés (béliers mâles castrés de race Texel âgés de I 8 mois au départ et pesant 40 à $50 \mathrm{~kg}$ ) ont été les mêmes pour l'étude d'une espèce végétale ou variété donnée au long du I $^{\text {er }}$ cycle et lors des cycles de repousses d'une même année. Le nombre de moutons utilisés n'est cependant pas suffisant pour tester correctenıent des différences éventuelles d'une espèce ou d'une variété à l'autre. Aussi, avons-nous étudié essentiellement les modifications du comportement suivant l'âge du fourrage distribué indépendamment de l'espèce végétale. Les différences liées à l'espèce végétale ne seront signalées que si clles sont importantes.

Les fourragcs verts ont été fauchés chaque matin, hachés dans un hache-paille (brins de 2 à $3 \mathrm{~cm}$ ) et distribués ad libitum en 3 repas par jour à $8 \mathrm{~h} 30,13 \mathrm{~h} 30 \mathrm{ct}$ I 7 heures; les quantités offertes ont été pesées à chaque distribution, les refus journaliers enlevés ct pesés avant la distribution du premier repas de la journée.

Les définitions des différents paramètres ont été données par Dulphy (I97I). Les durées journalières d'ingestion et de rumination correspondent à la somme de toutes les activités d'ingestion, de rumination durant 24 heures. Les durées unitaires d'ingestion et de rumination correspondent au temps passé à ingérer ou à ruminer par g de matière sèche ingéré par $\mathrm{kg}$ de poids métabolique; ces durées varient donc en sens inverse des vitesses d'ingestion ou de rumination. Nous avons appelé repas (ou période de rumination) une période d'ingestion (ou de rumination) d'une durée au moins égale ou supérieure à 7 minutes. Dès qu'une interruption d'activité à l'intérieur d'une période d'activité donnée excède 7 minutes, deux périodes sont comptées. La durée moyenne 


\begin{tabular}{|c|c|c|c|c|c|}
\hline & & \multirow{2}{*}{ N } & \multicolumn{3}{|c|}{ Composition de la matière sèche $(\%)$} \\
\hline & & & Cendres & Matières azotées & Cellulose brute \\
\hline Graminées & $\begin{array}{l}1^{\mathrm{er}} \text { cycle } \ldots \\
2^{\circ} \text { cycle } \ldots \ldots \\
3^{\mathrm{e}} \text { cycle } \ldots\end{array}$ & $\begin{array}{l}77 \\
45 \\
32\end{array}$ & $\begin{array}{ll}12,1 & (7,1-17,5) \\
11,8 & (8,2-16,1) \\
13,0 & (8,5-19,2)\end{array}$ & $\begin{array}{lr}12,7 & (5,7-26,7) \\
14,5 & (7,7-21,2) \\
15,3 & (10,4-19,2)\end{array}$ & $\begin{array}{l}28,3(18,6-37,9) \\
28,3(23,4-34,4) \\
24,7(20,9-24,8)\end{array}$ \\
\hline $\begin{array}{l}\text { Ray-grass } \\
\text { italien }\end{array}$ & $\left\{\begin{array}{l}1^{\mathrm{er}} \text { cycle } \ldots \\
2^{\mathrm{e}} \text { cycle } \ldots \ldots \\
3^{\mathrm{e}} \text { cycle } \ldots\end{array}\right.$ & $\begin{array}{r}7 \\
14 \\
7\end{array}$ & $\begin{array}{l}13,7 \quad(10,1-16,5) \\
11,0 \quad(8,2-15,8) \\
11,4 \quad(8,5-17,5)\end{array}$ & $\begin{array}{l}17,5(11,2-26,7) \\
14,8 \quad(9,9-21,2) \\
13,4(10,4-16,8)\end{array}$ & $\begin{array}{l}22,8(18,6-29,2) \\
28,5(23,4-30,7) \\
22,4(20,9-24,8)\end{array}$ \\
\hline Ray-grass at & glais $\ldots \ldots \ldots$ & 7 & $11,1(10,2-12,8)$ & $13,2 \quad(6,9-24,6)$ & $24,8(22,2-31,4)$ \\
\hline $\begin{array}{l}\text { Fétuque } \\
\text { élevée }\end{array}$ & $\left\{\begin{array}{l}1^{\text {er }} \text { cycle } \ldots \\
2^{\mathrm{e}} \text { cycle } \ldots \\
3^{\mathrm{e}} \text { cycle } \ldots\end{array}\right.$ & $\begin{array}{l}39 \\
11 \\
12\end{array}$ & $\begin{array}{l}12,8 \quad(9,9-17,5) \\
13,9 \quad(12,6-16,1) \\
14,4(12,6-17,2)\end{array}$ & $\begin{array}{l}12,9 \quad(7,8-21,1) \\
15,4(10,6-19,4) \\
15,5 \quad(12,7-18,8)\end{array}$ & $\begin{array}{l}28,2(20,5-35,8) \\
26,3(23,7-28,6) \\
25,3(23,6-27,6)\end{array}$ \\
\hline Dactyle & $\mid \begin{array}{l}1^{\text {er }} \text { cycle } \ldots \\
2^{\text {e }} \text { cycle } \ldots \\
3^{\text {e }} \text { cycle } \ldots .\end{array}$ & $\begin{array}{l}7 \\
7 \\
7\end{array}$ & $\begin{array}{l}12,3 \quad(9,5-15,1) \\
11,1 \quad(9,6-12,6) \\
11,7 \quad(12,4-19,2)\end{array}$ & $\begin{array}{l}14,0 \quad(9,9-24,3) \\
15,4(10,9-18,9) \\
16,0(12,4-19,2)\end{array}$ & $\begin{array}{l}25,5(20,1-33,3) \\
29,6(26,6-31,0) \\
25,6(23,6-27,1)\end{array}$ \\
\hline Fléole & $1^{\mathrm{er}}$ cycle $\ldots$ & 13 & $8,9 \quad(7,1-11,5)$ & $11,1 \quad(8,0-17,5)$ & $32,6(24,1-37,9)$ \\
\hline Brome & $\mid \begin{array}{l}1^{\text {er }} \text { cycle } \ldots \\
2^{\mathrm{e}} \text { cycle } \ldots\end{array}$ & $\begin{array}{r}10 \\
7\end{array}$ & $\begin{array}{ll}11,5 & - \\
11,0 & -\end{array}$ & $\begin{array}{ll}10,5 & (5,7-15,5) \\
10,5 & (7,7-13,5)\end{array}$ & $\begin{array}{l}30,3(26,9-36,0) \\
31,9(29,9-34,4)\end{array}$ \\
\hline $\begin{array}{l}\text { Trefle } \\
\text { violet }\end{array}$ & $\begin{array}{l}1^{\mathrm{er}} \text { cycle } \ldots \\
2^{\mathrm{e}} \text { cycle } \ldots\end{array}$ & $\begin{array}{r}18 \\
8\end{array}$ & $\begin{array}{l}12,6(10,0-16,0) \\
11,7(10,0-16,1)\end{array}$ & $\begin{array}{l}16,7(13,2-23,8) \\
19,2(16,6-27,4)\end{array}$ & $\begin{array}{l}24,2(17,0-32,3) \\
24,2(16,6-27,4)\end{array}$ \\
\hline
\end{tabular}

TABLEAU I $b$. - Digestibilité et ingestibilité des fourrages verts étudiés de 1966 à 1971

\begin{tabular}{|c|c|c|c|c|}
\hline \multicolumn{3}{|c|}{ Digestibilité } & \multirow{2}{*}{$\begin{array}{l}\text { Ingestibilité } \\
\left(g / k g P^{0,75}\right)\end{array}$} & \multirow{2}{*}{$\begin{array}{c}\text { Teneur en } \\
\text { matière sèche }(\%)\end{array}$} \\
\hline $\begin{array}{l}\text { Matières } \\
\text { organiques }\end{array}$ & $\begin{array}{l}\text { Matières } \\
\text { azotées }\end{array}$ & $\begin{array}{l}\text { Cellulose } \\
\text { brute }\end{array}$ & & \\
\hline $69,8(55,5-84,1)$ & $66,2(52,8-83,9)$ & $69,7(50,9-88,4)$ & $64,7(39,8-94,1)$ & $18,9(15,8-26,7)$ \\
\hline $69,2(59,5-75,0)$ & $70,1(57,3-79,3)$ & $68,5(51,7-78,7)$ & $70,7(52,4-88,0)$ & $20,1(15,7-27,0)$ \\
\hline $72,2(67,6-76,0)$ & $68,9(53,8-78,1)$ & $69,8(47,3-76,7)$ & $73,8(61,5-88,6)$ & $17,5(15,5-20,1)$ \\
\hline $78,2(71,3-8 / 4,1)$ & $74,1(63,5-83,9)$ & $77,9(66,7-88,4)$ & $75,6(65,3-94,1)$ & - \\
\hline $66,7(59,5-7 / 4,8)$ & $71,0(64,4-78,3)$ & $63,5(51,7-78,7)$ & $69,8(56,6-82,6)$ & - \\
\hline $72,4\left(68,{ }^{\prime}-76,0\right)$ & $64,4(53,8-7 \cdot 2,1)$ & $64,8(4,7,3-76,1)$ & $79,1(7-, 6-85,8)$ & - \\
\hline $76,0(70,0-78,8)$ & $63,9(50,3-77,6)$ & $75,7(72,7-79$, 像 & $71,7(52,8-83,7)$ & - \\
\hline $67,1(55,5-78,6)$ & $65,2(53,9-79,4)$ & $66,9(50,9-81,3)$ & $64,3(48,2-85,5)$ & $19,9(16,5-26,7)$ \\
\hline $69,0(60,8-73,7)$ & $70,5(57,3-79,3)$ & $69,7(59,6-73,4)$ & $73,0(67,2-81,6)$ & $19,8(16,7-24,7)$ \\
\hline $70,1(67,6-7 / 1,8)$ & $70,6\left(6^{\prime}, 9-74,7\right)$ & $71,5(68,3-76,6)$ & $72,4(61,5-83,7)$ & $18,1(15,5-20,1)$ \\
\hline $71,9(60,4-76,6)$ & $70,0(60,3-79,8)$ & $71,6(58,5-76,6)$ & $70,8(18,3-82,3)$ & 一 \\
\hline $68,4(65,4-71,2)$ & $69,0(58,3-75,7)$ & $71,1(67,4-75,2)$ & $76,3(63,3-88,0)$ & - \\
\hline $71,4(68,9-75,3)$ & $69,4(62,4-78,1)$ & $68,8(65,0-76,7)$ & $78,6(66,7-88,6)$ & - \\
\hline $68,4(61,2-77,8)$ & $62,7(52,8-74,5)$ & $69,{ }^{\prime}(62,5-77,0)$ & $60,6(45,3-86,6)$ & $18,5(15,8-25,1)$ \\
\hline $73,1(67,0-79,8)$ & $\overline{73,1}$ & 76,3 & $59,1(39,8-74,8)$ & $19,2(15,9-26,3)$ \\
\hline $70,7(65,0-75,0)$ & 66,9 & 75,3 & $60,9(52,4-71,2)$ & $22,9(20,0-27,0)$ \\
\hline $71,4(57,6-82,6)$ & $73,7(64,5-81,1)$ & $65,1(46,5 \cdot 80,7)$ & $75,6(6 \cdot 2,3-91,2)$ & $13,7(10,6-17,8)$ \\
\hline $68,4(63,6-75,8)$ & $72,6(68,0-79,3)$ & $50,1(16,5-53,9)$ & $83,3(73,2-95,2)$ & $17,9(13,6-24,3)$ \\
\hline
\end{tabular}




\begin{tabular}{|c|c|c|c|c|c|c|c|c|c|}
\hline \multirow{7}{*}{ 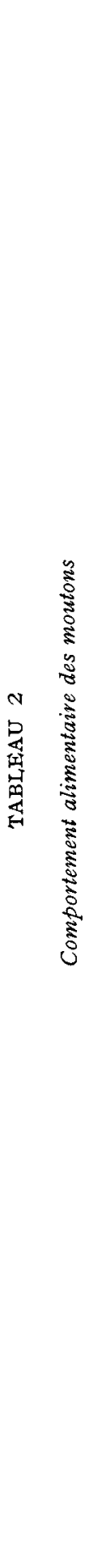 } & 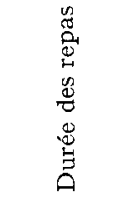 & 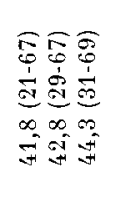 & 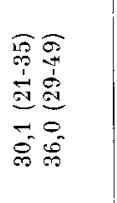 & 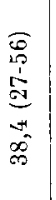 & 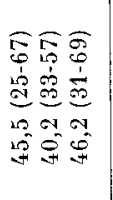 & 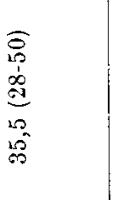 & 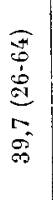 & 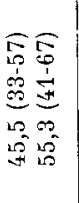 & 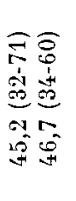 \\
\hline & 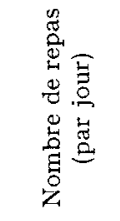 & 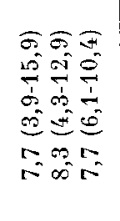 & 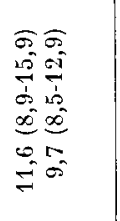 & 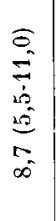 & 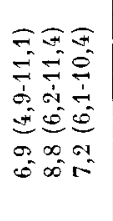 & 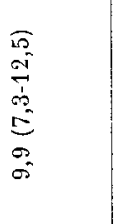 & 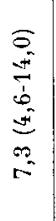 & 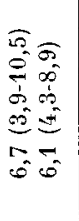 & 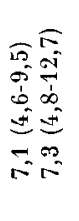 \\
\hline & 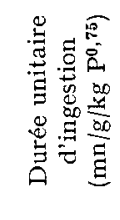 & 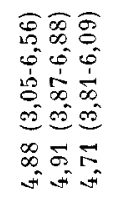 & 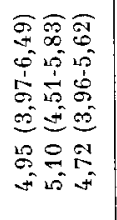 & 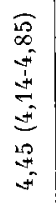 & 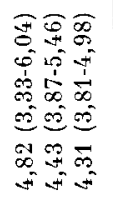 & 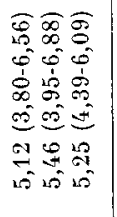 & 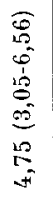 & 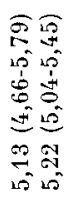 & 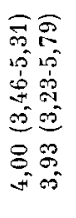 \\
\hline & 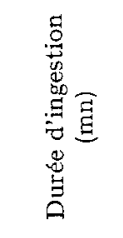 & 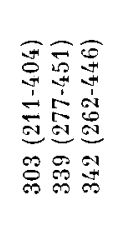 & 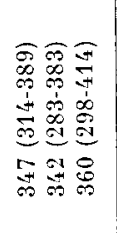 & 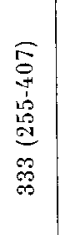 & 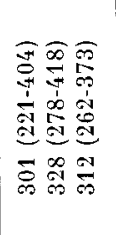 & 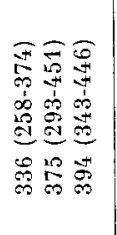 & 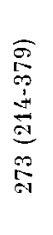 & 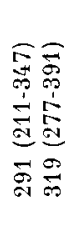 & 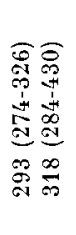 \\
\hline & 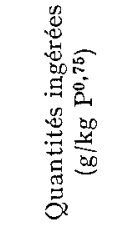 & 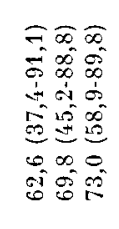 & 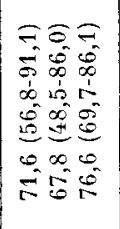 & $\begin{array}{l}\widehat{0} \\
0 \\
8 \\
b \\
0 \\
0 \\
0 \\
\infty \\
\infty \\
0 \\
0\end{array}$ & 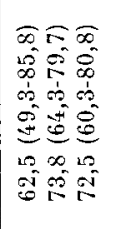 & 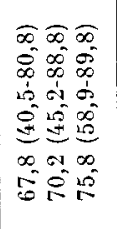 & $\begin{array}{l}0 \\
= \\
0 \\
0 \\
1 \\
1 \\
0 \\
0 \\
0 \\
0 \\
0 \\
0 \\
10\end{array}$ & 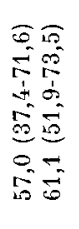 & 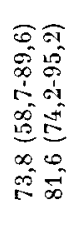 \\
\hline & & 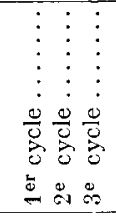 & $\begin{array}{ccc} & \vdots & \vdots \\
\vdots & \vdots & \vdots \\
\vdots & \vdots \\
0 & 0 \\
0 & 0 \\
0 & 0 \\
0 & 0 \\
0 & 0 \\
0 & 8 \\
0 & 0 \\
\end{array}$ & $\vdots$ & 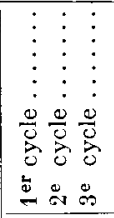 & 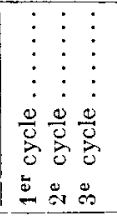 & $\begin{array}{c} \\
\vdots \\
\vdots \\
0 \\
0 \\
0 \\
5 \\
\end{array}$ & $\begin{array}{l}00 \\
0 \\
0 \\
0 \\
0 \\
0 \\
0 \\
0\end{array}$ & $\begin{array}{l}\vdots: \\
0 \\
0 \\
0 \\
0 \\
0 \\
0 \\
0 \\
0 \\
\end{array}$ \\
\hline & & 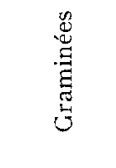 & 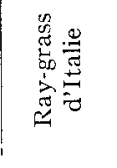 & 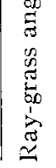 & 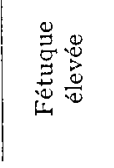 & $\begin{array}{l}\frac{0}{3} \\
\text { 总 } \\
\text { م. }\end{array}$ & $\frac{0}{\frac{0}{0}}$ & $\begin{array}{l}\text { घ̆ } \\
\text { ڤ్ }\end{array}$ & $\begin{array}{l}\text { 志 } \\
\text { 点 }\end{array}$ \\
\hline
\end{tabular}


des repas et périodes de rumination est obtenue en divisant les durées journalières d'ingestion ou de rumination par le nombre de périodes d'activité correspondantes. Nous appellerons en fin mastication l'ensemble des activités d'ingestion et de rumination.

\section{RÉSULTATS}

\section{Digestibilité et ingestibilité des fourrages étudiés}

Pour une espèce donnée, la digestibilité de la matière organique est liée au stade de végétation ou à 1'âge. Elle présente des valeurs élevées au début du $\mathbf{I}^{\text {er }}$ cycle, de l'ordre de 75 à 85 selon les espèces, puis diminue de façon curvilinéaire pour atteindre à la floraison des valeurs comprises entre 55 à 65 . La digestibilité des repousses est inférieure à celle des plantes du début du I $^{\text {er }}$ cycle, mais diminue moins rapidement avec l'âge.

Les fourrages ont été ingérés par les moutons en quantité très variable (tabl. 2). Pour une espèce donnée, les quantités ingérées diminuent constamment avec l'avancement du stade de végétation ou avec l'âge de la plante, en particulier au cours du I er cycle de végétation. Les quantités ingérées évoluent dans le même sens que la

TABLEAU 3

Coefficients de corrélation entre la digestibilité, les teneurs en matières azotées, en cellulose brute et les quantités ingérées

\begin{tabular}{|c|c|c|c|c|}
\hline & & $\begin{array}{l}\text { Digestibilité de la } \\
\text { matière organique }\end{array}$ & $\begin{array}{l}\text { Matières azotées tot. } \\
\text { (p. } 100 \text { MS) }\end{array}$ & $\begin{array}{l}\text { Cellulose brute } \\
\text { (p. } 100 \mathrm{MS} \text { ) }\end{array}$ \\
\hline Graminées & $\mid \begin{array}{ll}1 \text { er } & \text { cycle } \ldots . . . \\
2^{\text {e }} & \text { cycle } \ldots \ldots \\
3^{\text {e }} & \text { cycle } \ldots \ldots \\
\end{array}$ & $\begin{array}{r}+0,626 \\
+0,417 \\
-0,308\end{array}$ & $\begin{array}{l}+0,455 \\
+0,424 \\
\text { NS }\end{array}$ & $\begin{array}{c}-0,766 \\
-0,638 \\
\text { NS }\end{array}$ \\
\hline $\begin{array}{l}\text { Ray-grass } \\
\text { italien }\end{array}$ & $\mid \begin{array}{ll}1^{\mathrm{er}} & \text { cycle } \ldots \ldots \\
2^{\mathrm{e}} & \text { cycle } \ldots \ldots \\
3^{\mathrm{e}} & \text { cycle } \ldots \ldots \\
.\end{array}$ & $\begin{array}{l}\text { NS } \\
\text { NS } \\
\text { NS }\end{array}$ & $\begin{array}{l}\text { NS } \\
\text { NS } \\
\text { NS }\end{array}$ & 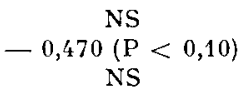 \\
\hline Ray-grass angla & $\ldots \ldots$ & $+0,820$ & $+0,852$ & $-0,866$ \\
\hline $\begin{array}{l}\text { Fétuque } \\
\text { élevée }\end{array}$ & $\left|\begin{array}{ll}1^{\mathrm{er}} & \text { cycle } \ldots \ldots \\
2^{\mathrm{e}} & \text { cycle } \ldots \ldots \\
3^{\mathrm{e}} & \text { cycle } \ldots \ldots \\
& \ldots \ldots\end{array}\right|$ & $\begin{array}{l}+0,640 \\
+0,66^{\prime} \\
\text { NS }\end{array}$ & $\begin{array}{l}+0,442 \\
\text { NS } \\
\text { NS }\end{array}$ & $\begin{array}{l}-0,653 \\
\text { NS } \\
\text { NS }\end{array}$ \\
\hline Dactyle & 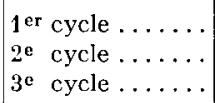 & $\begin{array}{l}+0,813 \\
\text { NS } \\
\text { NS }\end{array}$ & $\begin{array}{l}\text { NS } \\
\text { NS } \\
\text { NS }\end{array}$ & $\begin{array}{l}-0,873 \\
\text { NS } \\
\text { NS }\end{array}$ \\
\hline Fléole & $1^{\mathrm{er}}$ cycle $\ldots \ldots$ & $+0,916$ & $+0,776$ & $\longrightarrow 0,940$ \\
\hline Brome & $\begin{array}{l}\text { er } \\
2 \text { e cycle } \ldots \ldots\end{array}$ & $\begin{array}{l}+0,91 t_{t} \\
+0,91 t_{t}\end{array}$ & $\begin{array}{l}+0,722 \\
+0,896\end{array}$ & $\begin{array}{r}-0,822 \\
-0,772\end{array}$ \\
\hline Trèfle violet & $\begin{array}{l}1^{\text {er }} \text { cycle } \ldots \ldots \\
2 \text { e cycle } \ldots \ldots\end{array}$ & $\begin{array}{c}=+0,507 \\
\text { NS }\end{array}$ & $+0,450(\mathrm{P}<0,10)$ & $\begin{array}{l}\text { NS } \\
\text { NS }\end{array}$ \\
\hline
\end{tabular}


digestibilité et que la teneur en matières azotées et en sens inverse de la teneur en cellulose brute. Ces trois facteurs ont une influence différente selon les espèces ou le numéro du cycle (tabl. 3).

Les repousses sont en moyenne ingérées en quantités plus élevées que les plantes du I $^{\text {er }}$ cycle de digestibilité comparable, vraisemblablement parce que leurs teneurs en azote et en matière sèche sont plus élevées.

\section{Données comportementales}

\section{Durée journalière d'ingestion.}

La durée journalière d'ingestion (DI) varie dans de larges limites : de 2 Io à $450 \mathrm{mn}$ Elle est en moyenne de $303 \pm 48 \mathrm{mn}$ (2I p. Ioo du temps journalier), $339 \pm 47$ (23,5 p. Ioo du temps journalier) et $342 \pm 48 \mathrm{mn}(23,7 \mathrm{p}$. IOO) pour les fourrages du $\mathrm{I}^{\mathrm{er}}, 2^{\mathrm{e}}$ et $3^{\mathrm{e}}$ cycle de graminées et de $300 \pm 34 \mathrm{mn}(20,8 \mathrm{p}$. IOO) pour les trèfles violets.

Elle diminue généralement avec l'âge des fourrages, c'est-à-dire en même temps que la digestibilité (tab1. 4). Les liaisons trouvées ne sont pas toujours significatives car la diminution du temps d'ingestion est plus ou moins importante suivant les espèces ; c'est ainsi qu'au cours du $\mathrm{I}^{\text {er }}$ cycle quand la digestibilité passe de 80 à 60 p. Ioo, le temps d'ingestion diminue de $375-350$ à $25^{\circ} \mathrm{mn}$ pour la fétuque lutine, mais seulement de $375-35^{\circ}$ à $300-325$ pour le ray-grass italien Westervold. Par contre, elle ne varie pas pour le trèfle violet pour lequel elle reste comprise entre 300 et $325 \mathrm{mn}$.

De même que la durée d'ingestion évolue dans le même sens que la digestibilité, elle augmente avec la teneur en matières azotées et diminue lorsque la teneur en cellulose brute augmente.

Plus qu'à la digestibilité, la durée journalière d'ingestion est liée directement aux quantités ingérées de matière fraîche, de matière sèche, de cellulose brute et de matière organique digestible mais non à celles de matière organique non-digestible (tabl. 4).

Le trèfle violet, quoique ingéré en grande quantité, l'est cependant dans un temps plus court que les graminées.

Nombre et durée des repas par jour.

Le nombre de repas (nr) effectué par jour (y compris ceux qui ont suivi les 3 distributions journalières de fourrage) varie dans des proportions très importantes : de 3,9 à I5,9. Les moutons effectuent en moyenne 7,7 $\pm 2,6,8,3 \pm 2,3$ et 7,7 $\pm 1,9$ repas lorsqu'ils reçoivent les fourrages de graminées des $\mathrm{I}^{\mathrm{er}}, 2^{\mathrm{e}}$ et $3^{\mathrm{e}}$ cycles respectivement et 7,2 $\pm 1,9$ repas lorsqu'ils reçoivent les fourrages de trèfle violet. La durée moyenne des repas varie également beaucoup : de $2 \mathrm{I}$ à $69 \mathrm{mn}$; elle est en moyenne de $4 \mathrm{I}, 8 \pm \mathrm{II}, 6 \mathrm{mn}, 42,8 \pm \mathrm{I} 0,4$ et $44,3 \pm 9,6 \mathrm{mn}$ pour les graminées aux I ${ }^{\mathrm{er}}, 2^{\mathrm{e}}$ et $3^{\text {e }}$ cycles et de $45,7 \pm 12,5$ pour le trèfle violet.

Le nombre de repas diminue de façon importante avec l'âge des fourrages distribués, notamment au cours du $\mathrm{I}^{\mathrm{er}}$ cycle tandis que la durée moyenne des repas augmente. Notons qu'en moyenne au cours du I er cycle, le nombre de repas diminue de Io à 5 et leur durée passe de $30-35$ à $50 \mathrm{mn}$ lorsque la digestibilité du fourrage de graminées diminue de 80 à 60 p. Ioo alors que pour les $2^{\mathrm{e}}$ et $3^{\mathrm{e}}$ cycle aucune évolution 


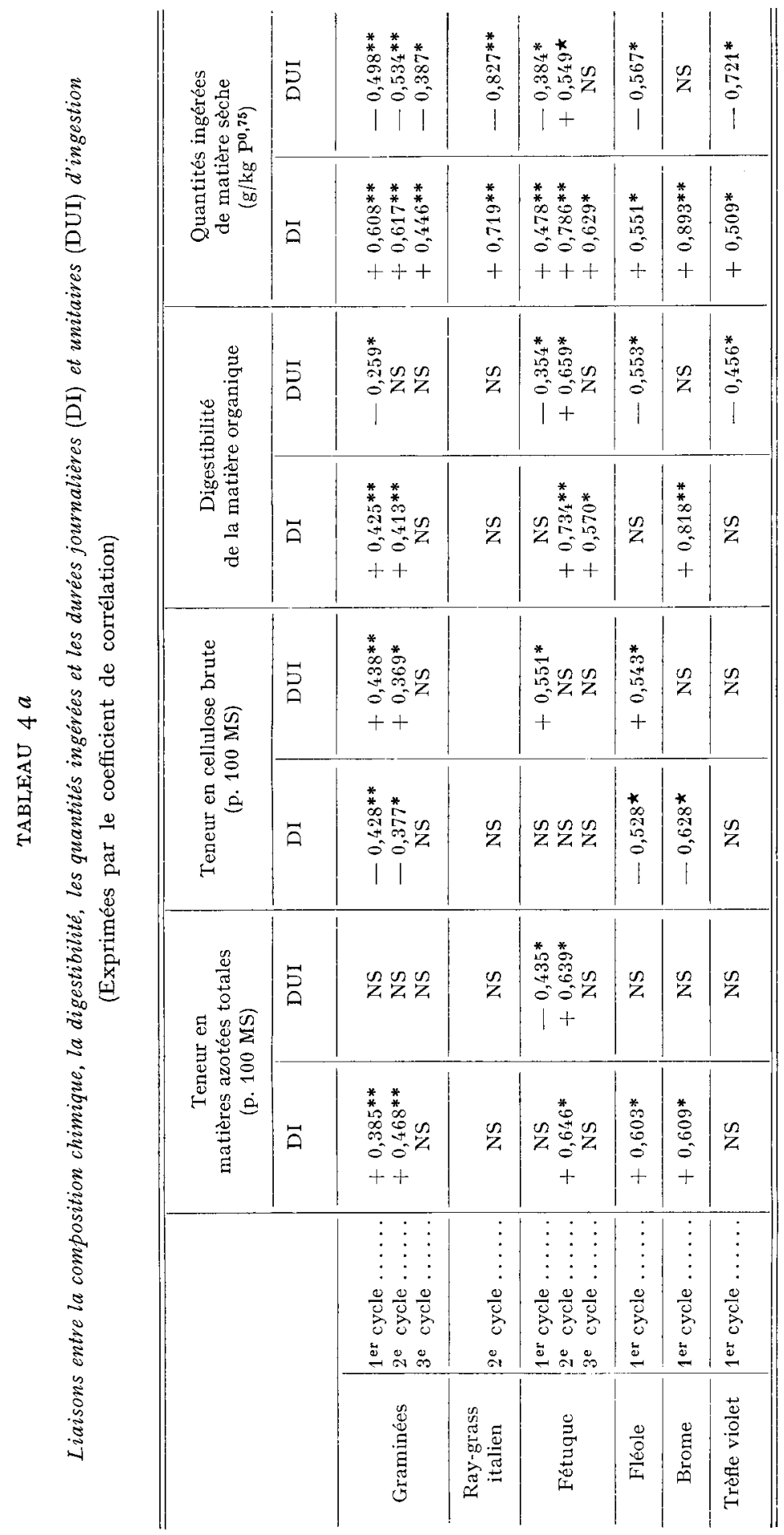




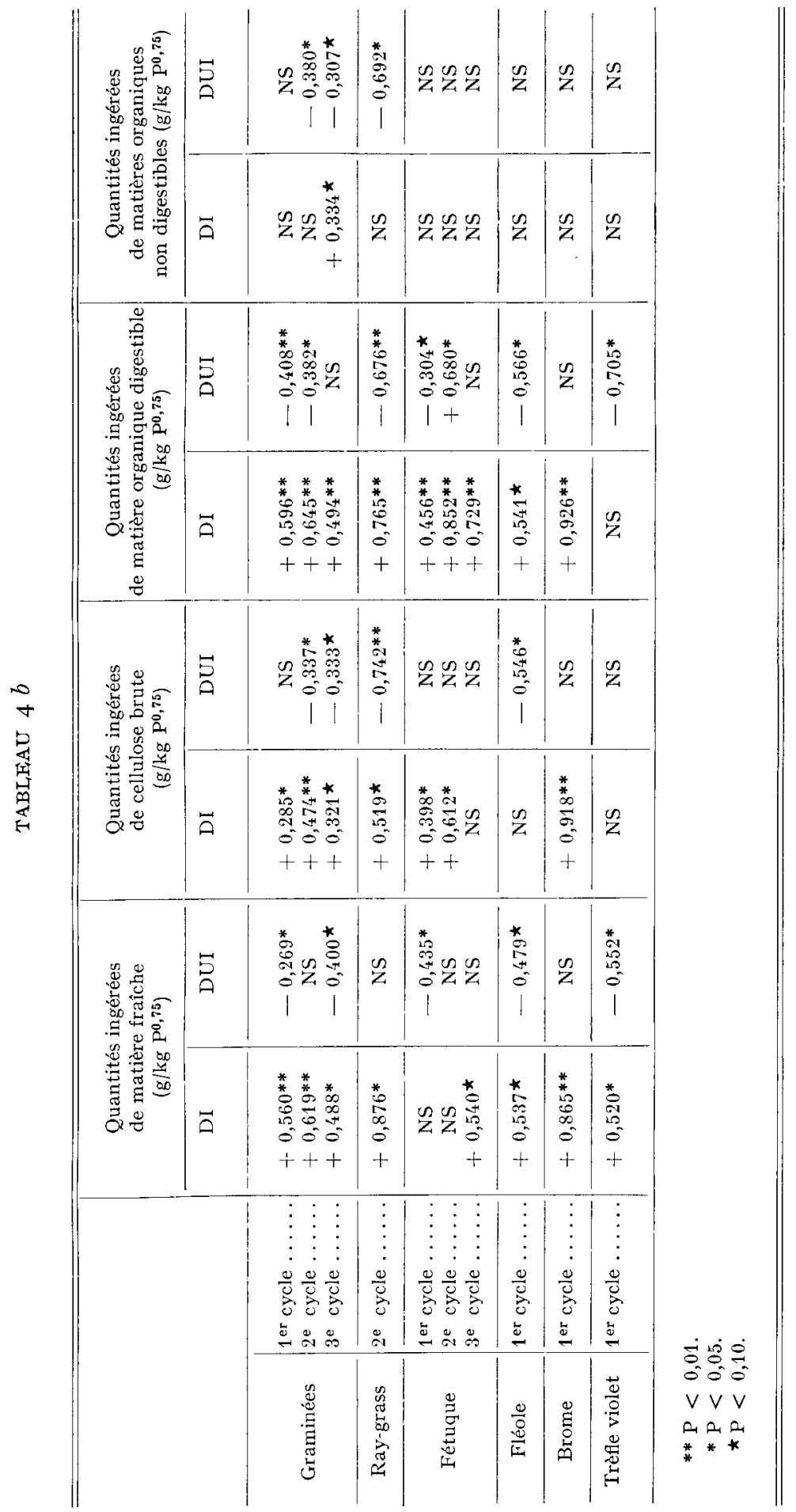


précise n'est notable. Les variations du nombre de repas sont cependant les plus nettes; elles sont directement liées à la digestibilité et à la teneur en matières azotées et inversement liées à la teneur en cellulose brute. Ces liaisons sont rapportées en détail dans le tableau 5 .

D'une manière générale, la diminution du nombre de repas explique en grande partie la diminution des quantités ingérées de matière sèche et surtout de matière fraîche ou de matière organique digestible. Simultanément, la durée moyenne des repas cst augmentée. Ce phénomène traduit essentiellement une diminution du nombre des petits repas (autres que ceux suivant les distributions de fourrages), mais il nous faudra préciser lors d'études ultérieures, 1'importance de ces petits repas.

\section{Lisirée unitaire d'ingestion.}

La durée unitaire d'ingestion est très variable suivant les échantillons étudiés : de 3,05 à $6,88 \mathrm{mn} / \mathrm{g}$ de $\mathrm{MS} / \mathrm{kg} \mathrm{P}^{0,75}$. Elle est en moyenne de 4,88 $\pm 0,72$, de 4,9I $\pm 0,64$ et cle $4,7 \mathrm{I} \pm 0,64$ pour les graminées respectivement au $\mathrm{I}^{\mathrm{er}}, 2^{\mathrm{e}}$ et $3^{\mathrm{e}}$ cycles de végétation et seulement de $3,98 \pm 0,55$ pour les trèfles violets.

Pour une espèce donnée, la durée unitaire d'ingestion varie peu avec le stade de croissance ou le numéro de cycle. Elle a cependant tendance à augmenter légèrement avec l'âge de la plante au cours du I er cycle de végétation, notamment chez les espèces pour lesquelles la durée journalière d'ingestion varie peu. C'est ainsi que pour le ray-grass italien Westervold elle augmente au cours du I er cycle passant de 4 à $5 \mathrm{mn} / \mathrm{g}$ de $\mathrm{MS} / \mathrm{kg} \mathrm{P}^{0,75}$; environ. Aussi, n'obtenons-nous qu'une liaison très lâche quoique significative avec la digestibilité et inexistante avec la teneur en matières azotées pour les fourrages du $I^{\text {er }}$ cycle (tabl. 4). Par contre, la durée unitaire d'ingestion augmente en même temps que la teneur en cellulose brute pour les premiers cycles de graminées.

De même, la durée unitaire d'ingestion varie peu avec les quantités ingérées, mais elle augmente un peu et souvent de façon significative quand les quantités de matière sèche et de matière organique digestible ingérées diminuent (tab1. 4).

Il est donc curieux de constater que la vitesse d'ingestion de la matière sèche d'une plante donnée est pratiquement indépendante de sa composition morphologique (présence ou absence de tige) et de son âge. Il est vrai cependant que tous les fourrages ont été distribués à l'auge après avoir été hachés dans un hache-paille, ce qui a dû diminuer notamment les différences de préhensibilité entre fourrages feuillus ou à tiges. Compte tenu du fait que la durée unitaire d'ingestion de la matière sèche varie peu ou augmente légèrement avec l'âge et que la teneur en matière sèche de la plante augmente, la durée unitaire d'ingestion de la matière fraîche diminue donc très notablement avec l'âge de la plante quand la digestibilité diminue.

En revanche, il existe des différences très nettes entre fourrages ; le trèfle violet est ingéré plus rapidement que les graminées, et le ray-grass anglais plus rapidement que le dactyle ou le brome par exemple : les différences entre espèces ne semblent pas liées à des différences dans leurs teneurs en azote ou en cellulose brute. Peut-être traduisent-elles des différences d'appétibilité suivant les plantes. Ces différences d'appétibilité sont peut-être dues aux teneurs en glucides ou autres constituants solubles ou bien à des différences entre les caractéristiques physiques de ces plantes, notamment au niveau de la structure des épidermes. 
(1)

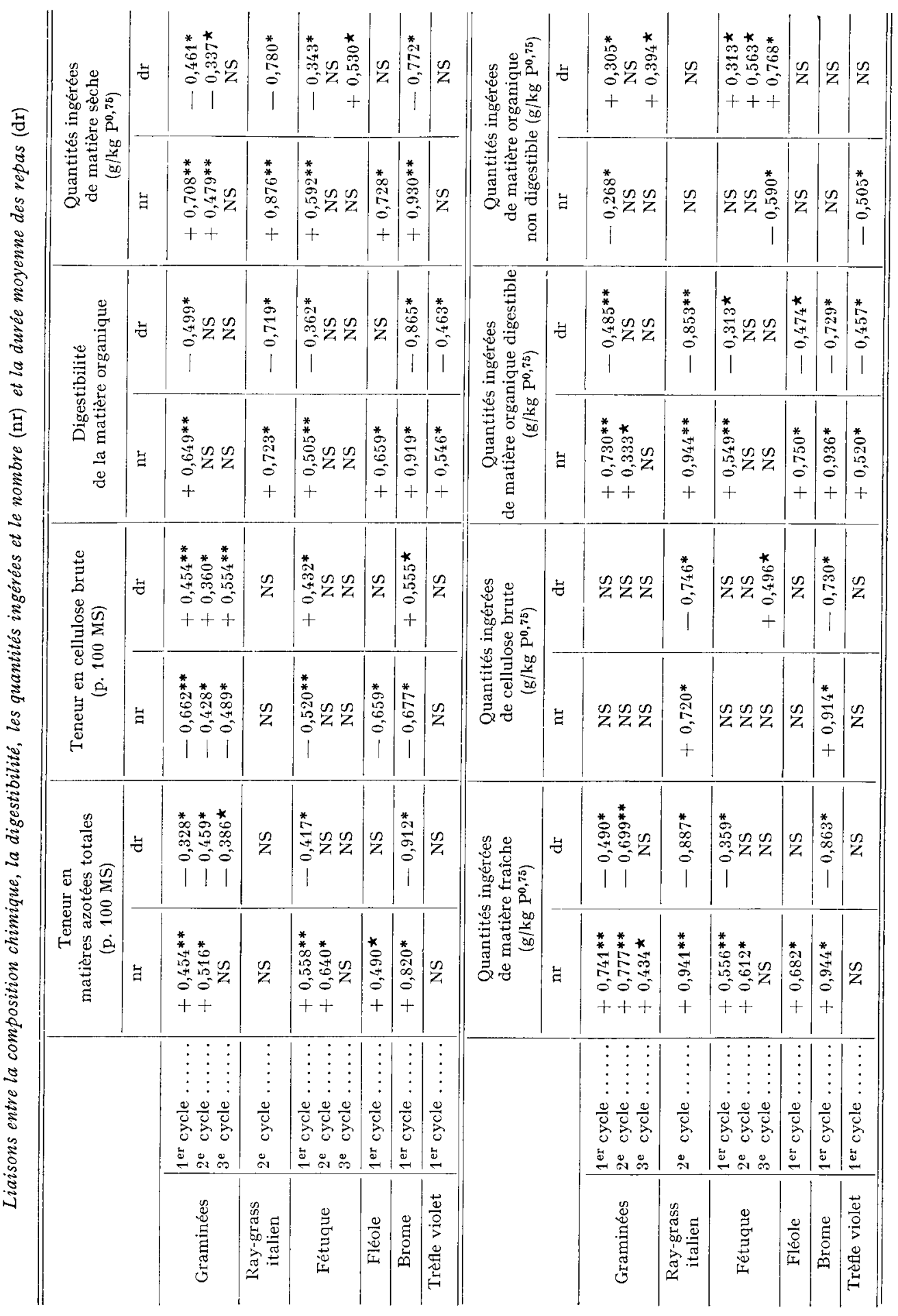


Durée journalière de rumination (tabl. 6).

Malgré quelques valeurs extrêmes éloignées (3I7 à $644 \mathrm{mn}$ ) la durée journalière de rumination (DR) varie beaucoup moins que la durée journalière d'ingestion. Elle est en moyenne respectivement de $529 \pm 66 \mathrm{mn}(36,7 \mathrm{p}$. Ioo du temps journalier), $528 \pm 62 \mathrm{mn}(35,6 \mathrm{p}$. Ioo du temps journalier), et $484 \pm 72$ (33,6 p. IоO du temps journalier) avec les fourrages de graminées du $\mathrm{I}^{\mathrm{er}}, 2^{\mathrm{e}}$ et $3^{\mathrm{e}}$ cycles de végétation et $497 \pm 88$ (34,5 p. Ioo du temps journalier) avec les trèfles violets.

\section{TABLEAU 6}

Comportement mérycique des moutons et mastication

\begin{tabular}{|c|c|c|c|c|c|}
\hline & & \multicolumn{2}{|c|}{ Rumination } & \multicolumn{2}{|c|}{ Mastication } \\
\hline & & $\underset{(\mathrm{mn})}{\text { Durée journalière }}$ & $\begin{array}{l}\text { Durée unitaire } \\
\left(\mathrm{mn} / \mathrm{g} / \mathrm{kg} \mathrm{P}^{\top}, 75\right)\end{array}$ & Durée journalière & $\begin{array}{l}\text { Durée unitaire } \\
\left(\mathrm{mn} / \mathrm{g} / \mathrm{kg} \mathrm{P}^{0,75}\right)\end{array}$ \\
\hline Graminées & $\left\{\begin{array}{lll}1 \text { er } & \text { cycle } & \ldots \\
2^{\mathrm{e}} & \text { cycle } & \ldots \\
3^{\mathrm{e}} & \text { cycle } & \ldots\end{array}\right.$ & $\begin{array}{l}529(317-663) \\
528(419-636) \\
484(342-609)\end{array}$ & $\begin{array}{l}8,74(4,51-11,94) \\
7,75(4,53-11,89) \\
6,68(4,85-8,82)\end{array}$ & $\begin{array}{l}832(686-974) \\
868(721-971) \\
817(536-960)\end{array}$ & $\begin{array}{l}13,63(9,15-18,92) \\
12,68(9,00-16,92) \\
11,39(9,21-13,26)\end{array}$ \\
\hline $\begin{array}{l}\text { Ray-grass } \\
\text { italien }\end{array}$ & $\left|\begin{array}{lll}1^{\mathrm{er}} & \text { cycle } & \ldots \\
2^{\mathrm{e}} & \text { cycle } & \ldots \\
3^{\mathrm{e}} & \text { cycle } & \ldots\end{array}\right|$ & $\begin{array}{l}451(317-554) \\
559(464-636) \\
494(400-581)\end{array}$ & $\begin{array}{l}6,36(5,58-8,45) \\
8,38(6,87-11,89) \\
6,46(5,45-7,73)\end{array}$ & $\begin{array}{l}799(686-876) \\
902(790-971) \\
808(536-925)\end{array}$ & $\begin{array}{l}11,31(9,26-13,36) \\
13,47(11,44-14,80) \\
11,17(9,96-13,26)\end{array}$ \\
\hline Ray-grass a & nglais & $506(389-597)$ & $7,19(1,53-11,35)$ & $839(712-918)$ & $11,72(9,00-16,20)$ \\
\hline Fétuque & $\left|\begin{array}{lll}1^{\mathrm{er}} & \text { cycle } & \ldots \\
2^{\mathrm{e}} & \text { cylce } & \ldots \\
3^{\mathrm{e}} & \text { cycle } & \ldots\end{array}\right|$ & $\begin{array}{l}544(387-663) \\
554(481-618) \\
525(417-609)\end{array}$ & $\begin{array}{l}8,86(4,51-11,33) \\
7,52(6,77-8,55) \\
7,26(5,57-8,82)\end{array}$ & $\begin{array}{l}845(700-974) \\
883(756-956) \\
837(763-960)\end{array}$ & $\begin{array}{l}13,69(9,15-17,70) \\
11,94(10,71-13,08) \\
11,57(10,20-13,19)\end{array}$ \\
\hline Dactyle & $\left|\begin{array}{lll}1^{\mathrm{er}} & \text { cycle } & \ldots \\
2^{\mathrm{e}} & \text { cycle } & \ldots \\
3^{\mathrm{e}} & \text { cycle } & \ldots\end{array}\right|$ & $\begin{array}{l}472(403-564) \\
452(119-486) \\
418(342-479)\end{array}$ & $\begin{array}{l}7,31(5,64-11,65) \\
6,75(5,22-10,04) \\
5,56(4,85-6,58)\end{array}$ & $\begin{array}{l}808(730-866) \\
832(798-902) \\
811(701-919)\end{array}$ & $\begin{array}{l}12,43 \\
12,22(\mathbf{9}(9,16-18,02) \\
10,81(9,21-12,62)\end{array}$ \\
\hline Fléole & $1^{\text {er }}$ cycle & $557(452-636)$ & $9,92(5,23-11,54)$ & $831(705-921)$ & $14,67(9,61-18,45)$ \\
\hline Brome & $\left|\begin{array}{lll}1^{\text {er }} & \text { cycle } & \ldots \\
2^{\text {e }} & \text { cycle } & \cdots\end{array}\right|$ & $\begin{array}{l}522(492-561) \\
546(478-574)\end{array}$ & $\begin{array}{l}9,49(7,16-11,94) \\
9,11(6,71-10,71)\end{array}$ & $\begin{array}{l}812(708-885) \\
865(823-9 ; 5)\end{array}$ & $\begin{array}{l}14,63(1,200-18,92) \\
14,18(11,75-16,16)\end{array}$ \\
\hline $\begin{array}{l}\text { Trèfle } \\
\text { violet }\end{array}$ & $\mid \begin{array}{l}1^{\text {er }} \text { cycle } \\
2^{\text {e }} \text { cycle }\end{array}$ & $\begin{array}{l}485(270-567) \\
523(360-594)\end{array}$ & $\begin{array}{l}6,72(3,66-9,48) \\
6,17(4,85-7,80)\end{array}$ & $\begin{array}{l}777(556-867) \\
841(790-874)\end{array}$ & $\begin{array}{c}10,71(7,12-14,03) \\
10,10(8,62-11,50)\end{array}$ \\
\hline
\end{tabular}

Elle augmente avec l'âge des fourrages, notamment au cours du premier cycle, mais de façon très variable suivant les plantes : de 300 à $55^{\circ} \mathrm{mn}$ par exemple pour le trèfle violet, seulement de $45^{0}-475$ à $55^{0}-600 \mathrm{mn}$ pour les fétuques élevées, et elle reste pratiquement constante (environ $525 \mathrm{mn}$ ) pour le brome. Ces évolutions différentes suivant les plantes semblent liées en grande partie à leur teneur en cellulose brute. En effet, la durée de rumination augmente en même temps que la teneur en cellulose brute jusqu'à ce que celle-ci atteigne une valeur voisine de 30 p. roo. Elle reste ensuite sensiblement constante, autour d'une valeur comprise entre 525 et 
575 minutes suivant la plante ou l'année, valeur qui semble dépendre plus du lot de moutons utilisés que de la plante. C'est ce qu'illustre la figure $I$, dans laquelle nous avons porté la durée de rumination en fonction de la teneur en cellulose brute des plantes du I $^{\text {er }}$ cycle pour lesquelles nous disposions d'un nombre suffisant de données. Ces données sont en outre regroupées par classe de teneur en cellulose brute (teneurs $<20$, comprises entre 20 et 24,24 et 28,28 et $32,>32$ ) pour que les valeurs obtenues soient plus représentatives. Ia durée de rumination augmente donc avec l'âge du fourrage et par conséquent est inversement liée à la digestibilité de la plante ou à sa teneur en matières azotées (tabl. 7) que pour les plantes dont la teneur en cellulose brute était encore très largement inférieure à $30 \mathrm{p}$. Ioo au stade où leur étude a démarré.

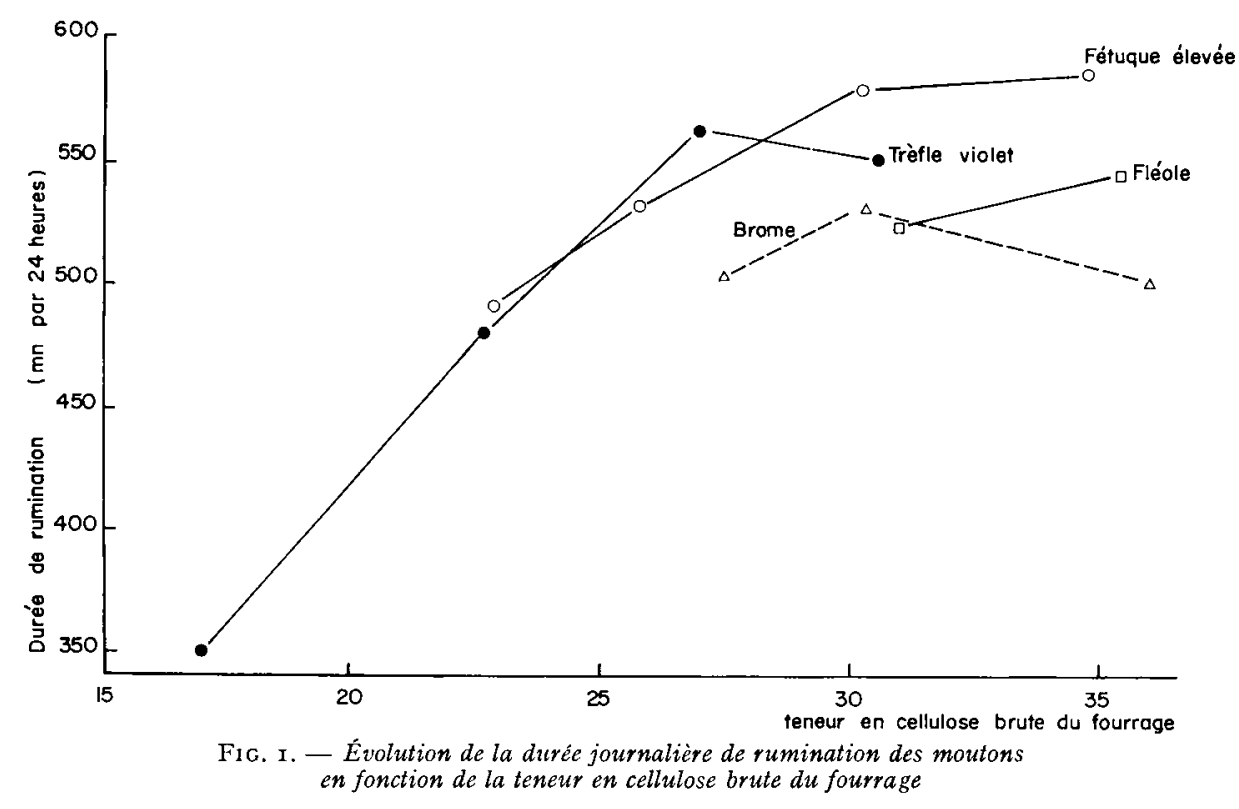

A l'inverse de la durée d'ingestion, la durée de rumination n'est pratiquement pas liée aux quantités de matière sèche ou de matière fraîche ingérées. En revanche, elle varie souvent dans le même sens que les quantités de cellulose brute ou de matière organique non-digestibles ingérées (tab1. 7).

Durée unitaire de rumination.

La durée unitaire de rumination (DUR) varie dans des limites très larges : de $4,5 \mathrm{I}$ à $\mathrm{I} 2,55 \mathrm{mn} / \mathrm{g} / \mathrm{kg} \mathrm{P} \mathrm{P}^{0,75}$. Elle est en moyenne de $8,74 \pm 2,0 \mathrm{r}, 7,75 \pm \mathrm{I}, 59$ et $6,68 \pm \mathrm{I}, 07$ pour les graminées du $\mathrm{I}^{\mathrm{er}}, 2^{\mathrm{e}}$ et $3^{\mathrm{e}}$ cycles de végétation et de $6,64 \pm \mathrm{I}, 57$ pour les trèfles violets.

Elle augmente très nettement avec l'âge des fourrages, notamment au cours du I $^{\text {er }}$ cycle de végétation. Elle est donc également inversement liée à la digestibilité et à la teneur en matières azotées et directement liée à la teneur en cellulose brute. Ces liaisons souvent étroites sont rapportées au tableau. 7 . 


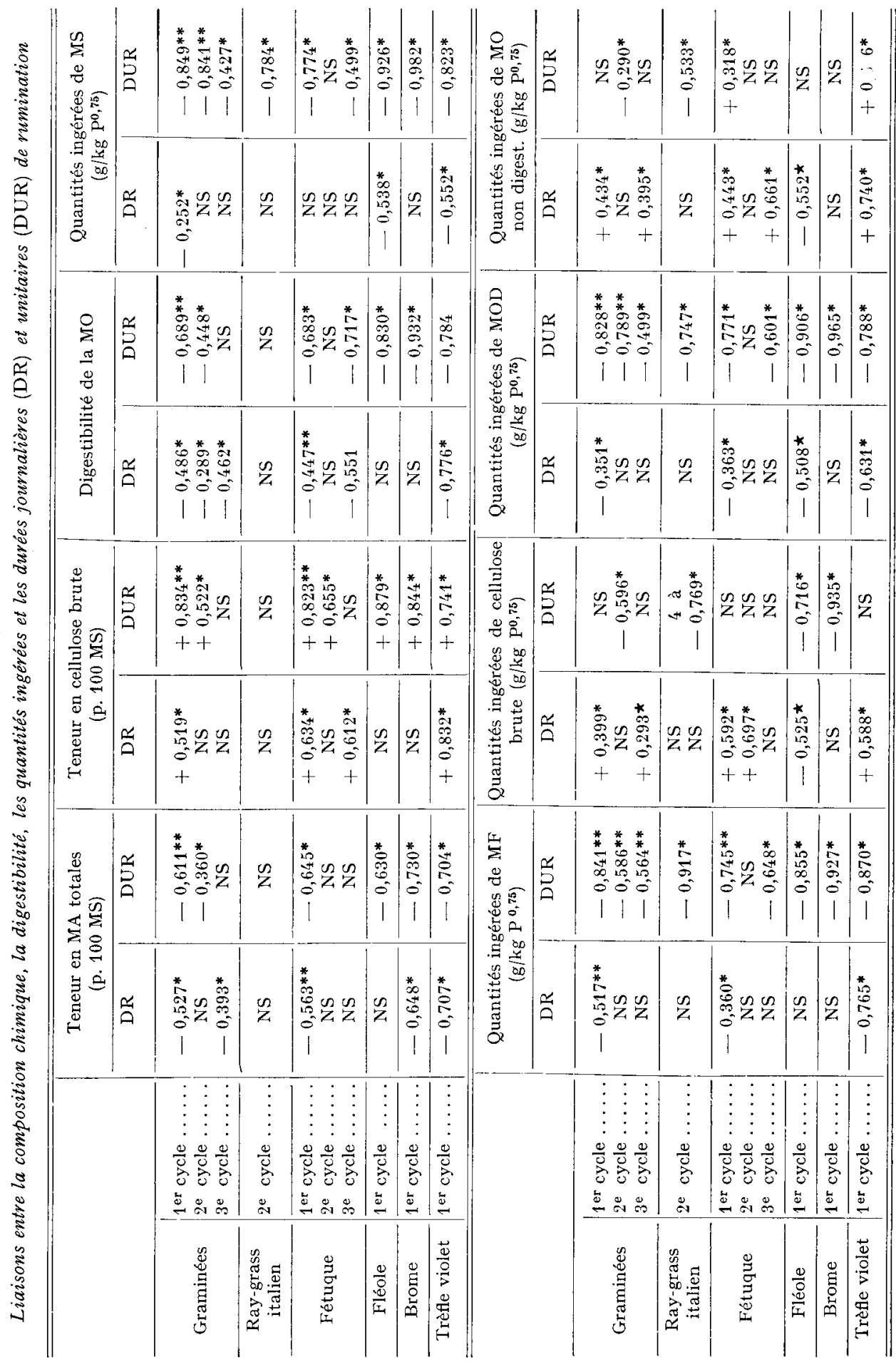


La durée unitaire de rumination est également inversement liée aux quantités de matière sèche, de matière fraîche et de matière organique digestible ingérées. Enfin, alors qu'il existe entre plantes de même digestibilité des différences importantes dans la durée unitaire de rumination, il n'en existe pratiquement plus à même quantité ingérée.

\section{Nombre et durée des périodes de rumination.}

Le nombre de périodes de rumination est peu variable d'une espèce à l'autre ou d'un cycle à l'autre. Il est en moyenne respectivement de $I 6,6 \pm 2,2, I 5,9 \pm I, 6$ et $\mathrm{I} 6,2 \pm 2,4$ pour les graminées du I ${ }^{\mathrm{er}}, 2^{\mathrm{e}}$ et $3^{\mathrm{e}}$ cycle de végétation et de $15,5 \pm 2,0$ pour les trèfles violets. La durée de ces périodes est légèrement plus variable: respectivement $32,6 \pm 6,5 \mathrm{mn}, 34,7 \pm 5,0$ et $32,9 \pm 7,8 \mathrm{mn}$ pour les graminées et $29,8 \pm 7, \mathrm{I}$ pour les trèfles violets.

Le nombre de périodes de rumination a parfois tendance à diminuer lorsque l'âge du fourrage augmente au premier cycle de croissance, en particulier pour les fétuques élevées et les fléoles. Par contre, leur durée augmente généralement, notamment pour les plantes telles que le trèfle violet ou le ray-grass italien Westervold pour lesquelles la durée journalière de rumination augmente considérablement avec l'âge de la plante ; c'est ainsi qu'au cours du I er cycle la durée moyenne des périodes passe de 20 à $40 \mathrm{mn}$ environ pour ces deux plantes.

Le nombre de périodes de rumination ne varie pratiquement pas avec la composition chimique de la plante mais augmente avec les quantités de matière sèche, de matière fraîche ou de matière organique digestible ingérées pour les fétuques et les fléoles au I er cycle et le ray-grass italien au second cycle. Par contre, la durée de ces périodes augmente avec la teneur en cellulose brute des plantes; elle diminue parfois avec les quantités de matière sèche, de matière fraîche et de matière organique digestible ingérées et augmente avec les quantités de cellulose brute ou de matière organique indigestible ingérées.

\section{Durée journalière de mastication (tab1. 6).}

La durée journalière de mastication (DM $=\mathrm{DI}+\mathrm{DR})$ est le paramètre le plus constant du comportement alimentaire. Cela est normal puisque, quand la plante vieillit, le temps d'ingestion et la durée de rumination évoluent en sens inverse. Elle est en moyenne de $832 \pm 70 \mathrm{mn}(57,8 \mathrm{p}$. Ioo du temps journalier $), 868 \pm 68 \mathrm{mn}$ $\left(60,2\right.$ p. roo) et $8 \mathrm{I} 7 \pm 89 \mathrm{mn}\left(56,7 \mathrm{p}\right.$. Ioo) pour les graminées au $\mathrm{I}^{\mathrm{er}}, 2^{\mathrm{e}}$ et $3^{\mathrm{e}}$ cycles de végétation et de $797 \pm 82 \mathrm{mn}(55,3 \mathrm{p}$. Ioo du temps journalier) pour les trèfles violets.

La durée de mastication évolue cependant un peu avec l'âge des fourrages mais de façon différente suivant les plantes puisqu'elle augmente avec la digestibilité pour le dactyle et le brome au $\mathrm{I}^{\mathrm{er}}$ cycle et diminue pour la fétuque et le trèfle (tab1. 8).

D'autre part, la durée de mastication varie peu avec les quantités ingérées. Cependant, on note des variations dans le même sens que les quantités de cellulose brute ingérées pour les fétuques, le brome et à moindre degré le trèfle violet.

En définitive, la durée de mastication est peu variable avec l'âge ou le numéro du cycle de végétation pour une plante donnée, et, par là, est pratiquement indépendante de la digestibilité et de la quantité ingérée. Elle a parfois tendance à augmenter légèrement au cours du premier cycle, en particulier pour le trèfle violet et le ray-grass 


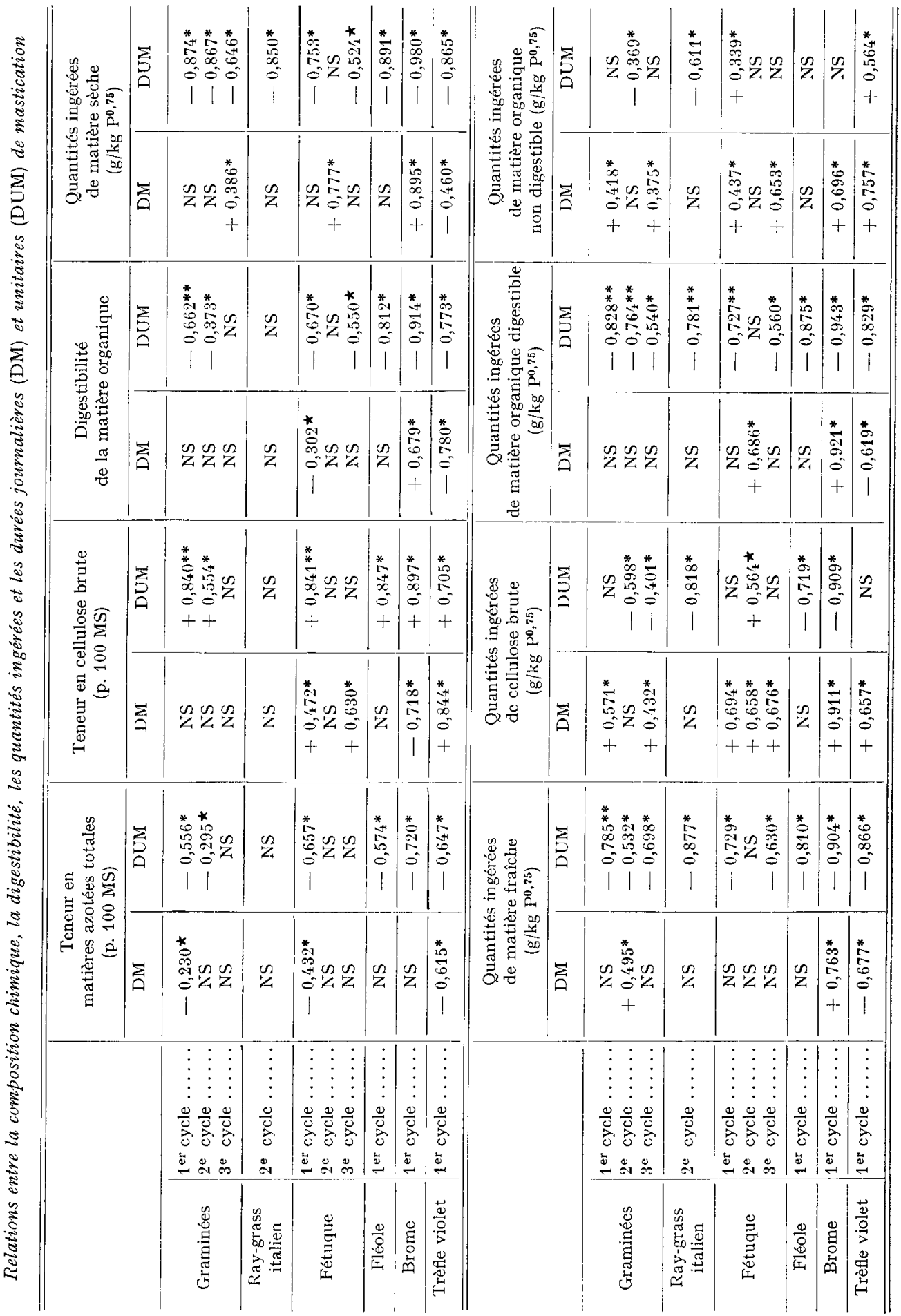


italien essentiellement à cause de l'augmentation du temps de rumination. Quelle que soit la plante et son stade d'exploitation, la durée de mastication se situe en général toujours entre 850 et $950 \mathrm{mn}$; les différences observées entre plantes, ou entre années pour une plante donnée, résultent peut-être plus, du lot de moutons employé que de la plante elle-même.

\section{Durée unitaire de mastication.}

La durée unitaire de mastication (DUM $=$ DUI + DUR), contrairement à la durée journalière de mastication est très variable : de 6,56 à $I 9,5 \mathrm{I} \mathrm{mn} / \mathrm{g} / \mathrm{kg} \mathrm{P}^{0,75}$. Des liaisons souvent étroites existent entre ce paramètre et la composition chimique pour toutes les espèces : évolution dans le même sens que la teneur en cellulose brute et en sens contraire à la teneur en matières azotées. I a durée unitaire de mastication est très étroitement inversement liée aux quantités de matière sèche, de matière fraîche, de matière organique digestible et dans une moindre mesure, de cellulose brute, ingérées (tabl. 8).

\section{DISCUSSION}

Lorsque la plante vieillit, notamment au cours du $\mathrm{I}^{\mathrm{e}}$ cycle de végétation, la quantité volontairement ingérée par le moutons diminue et nous observons parallèlement une modification du comportement alimentaire et mérycique de ce dernier.

Le nombre de repas diminue très nettement et il en est de même, mais dans une moindre mesure, de la durée journalière d'ingestion. Curieusement la durée unitaire d'ingestion varie peu; elle est donc pratiquement indépendante de la présence ou non de tiges. Cela n'est pas dû au fait que les fourrages ont été distribués après hachage puisque par ailleurs nous n'avons observé que de faibles différences de vitesse d'ingestion entre des fourrages verts hachés ou non (DULPHY, résultats nonpubliés).

En revanche, la durée journalière de rumination qui est faible lorsque les fourrages sont jeunes, pauvres en membranes (cellulose brute) et très digestibles, augmente rapidement, en même temps que la teneur en cellulose brute, jusqu'à une valeur voisine de Io heures et reste alors constante. BUTTERWORTH (I965) avait déjà observé ce phénomène : la durée de rumination chez le Mouton recevant du napier s'élevait de 7,9 à 9,8 heures au fur et à mesure que la plante vieillissait. De plus, la durée unitaire de rumination augmente de façon très importante (de I à 3 environ pour certains fourrages) elle est liée étroitement à la quantité ingérée et varie en sens inverse. Enfin, il est remarquable de constater que la durée journalière de mastication est un paramètre relativement constant, variant entre 850 et $950 \mathrm{mn}$ par jour, sauf pour les fourrages jeunes. La durée unitaire de mastication est également reliée étroitement à la quantité ingérée et varie en sens inverse. JourNET (résultats non-publiés) observe aussi sur des vaches laitières une liaison inverse entre la durée de rumination et la digestibilité de la ration d'une part, et entre la durée unitaire de mastication et la quantité ingérée d'autre part.

Comment peut-on expliquer ces modifications du comportement alimentaire et mérycique avec le stade de végétation du fourrage reçu par l'animal ? 
On sait que la quantité de fourrage ingérée par les ruminants est dans un premier temps réglée par des phénomènes de nature physique au niveau du rumen et du réseau (cf. Revue de Balch et Campling, I962; Demarquility, I965; Jarkige, DeMARQUiliy et DulPHY, I973). Plus précisément l'ingestibilité d'un fourrage est surtout déterminée par la quantité de particules alimentaires (en cours de digestion ou déjà digérées) présente, en moyenne, dans le rumen de l'animal. Elle dépend donc de la vitesse de vidange du rumen (cf schéma I) et par là, de la vitesse de digestion de la fraction digestible et de la vitesse de transit de la fraction indigestible. Ia vitesse de digestion est, à coup sûr, le facteur prépondérant puisque la digestion des tissus digestibles (parenchyme) est nécessaire à la libération des tissus indiges-

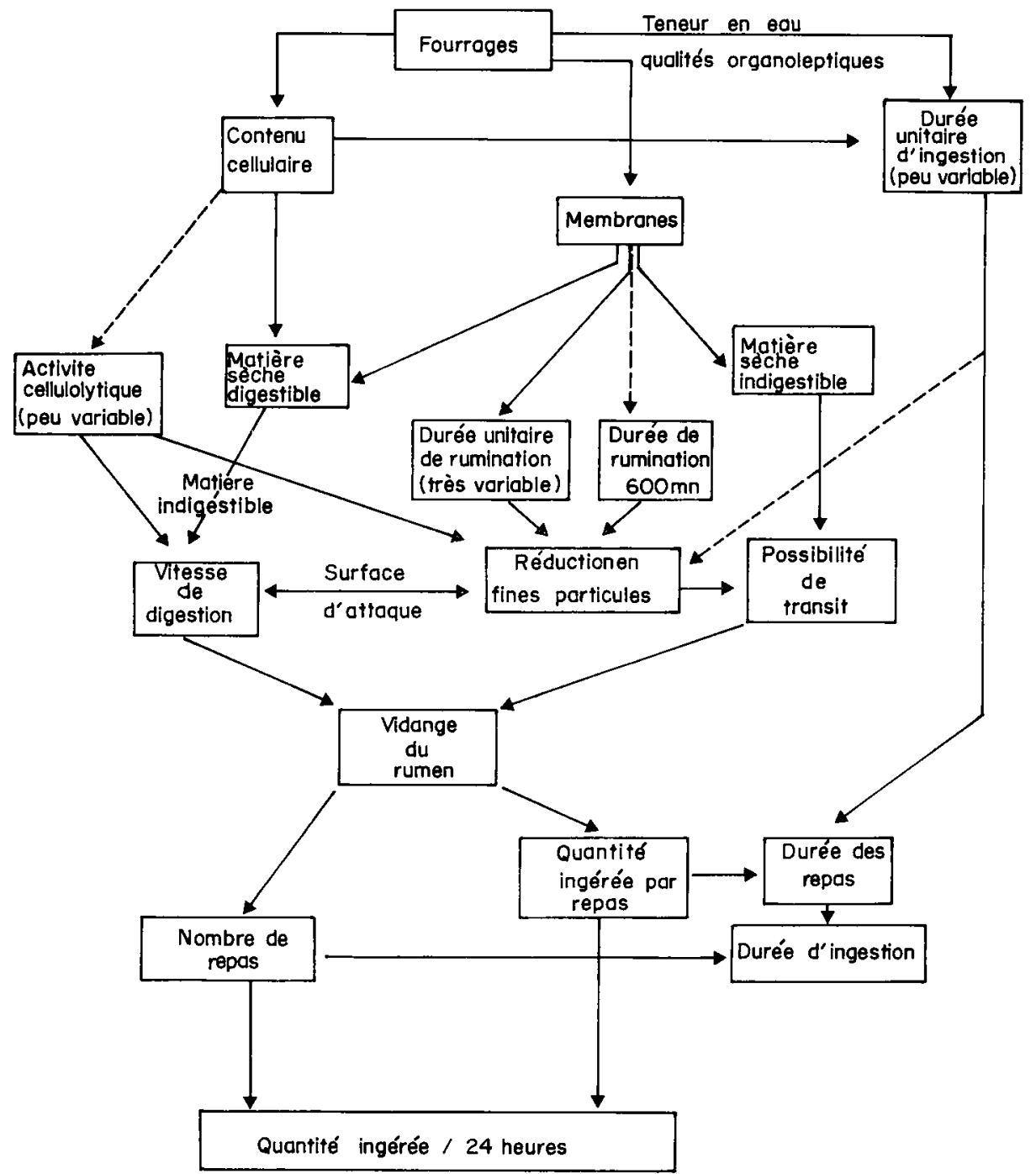

Schema I. - Liaisons entre phénomènes digestifs et manifestations du comportement alimentaire et mérycique 
tibles (sclérenchyme, vaisseaux...) et à leur réduction en particules suffisamment fines pour franchir l'orifice du feuillet. La vitesse de digestion dépend à son tour en partie de l'activité cellulolytique de la population microbienne du rumen (conditions du milieu, apport nutritif) et surtout de la résistance que lui offrent les tissus et les membranes (proportion de membranes, lignification de celles-ci...) ainsi que de la surface d'attaque, c'est-à-dire de la taille des particules. La mastication mérycique dont une des conséquences est la réduction de la taille des particules du contenu de rumen permet une augmentation des quantités ingérées, non seulement par une diminution de la place occupée par ces particules (FRENS, I960; BALCr et CAMPIING, I962) mais aussi et surtout en accélérant la vitesse de digestion (augmentation de la surface d'attaque). Elle est responsable aussi de la division des matériaux fibreux indigestibles (PEARCE et MoIr, I964; PEarce, I967; J. Blain, Boivin et BoST, I97I) favorisant ainsi leur possibilité de transit vers le feuillet. Il suffit d'ailleurs de diminuer son efficacité, par exemple en introduisant dans le rumen des fibres de polypropylène, pour abaisser considérablement la quantité de foin ingérée par les animaux (WELCH, rg67). La seule mastication mérycique à vide modifie en outre le transit des aliments par l'orifice réticulo-omasal (RUCKEBUSCH et LAPLACE, I967).

Avec les fourrages jeunes, pauvres en membranes, elles-mêmes peu lignifiées et très digestibles, la vitesse de digestion est élevée, la réduction en fines particules aisée. La durée unitaire de rumination et la durée journalière de rumination sont donc faibles, la vidange du rumen rapide, les repas nombreux et la quantité ingérée élevée. Notons à ce sujet que la durée unitaire de rumination doit être un excellent critère du temps nécessaire pour réduire une quantité de matière sèche donnée en particules suffisamment fines pour quitter le rumen.

Au fur et à mesure que le fourrage vieillit, la résistance des tissus et membranes à la digestion et à la réduction en fines particules s'élève; la vitesse de digestion diminue et la durée unitaire de rumination augmente. Au début cela peut être compensé, du moins en partie, par une augmentation parallèle du temps journalier passé à ruminer mais quand celui-ci atteint une valeur voisine de Io heures par jour, il se stabilise et reste alors constant. Il en résulte une vidange de moins en moins rapide du rumen, d'où une diminution progressive du nombre de repas et de la quantité ingérée.

En revanche, il ne nous est pas possible d'expliquer les différences de comportement alimentaire et mérycique chez les animaux recevant des plantes différentes. Il aurait fallu pour cela une étude beaucoup plus précise du comportement et surtout un nombre de moutons beaucoup plus important, compte tenu de la variabilité entre animaux sur un régime donné (DULPHy, I97I).

\section{Reçu pour publication en février 1974.}

\section{SUMMARY}

\section{FEEDING BEHAVIOUR AND RUMINATION IN SHEEP RECEIVING CHOPPED GREEN FORAGES}

Within the series of studies made on the voluntary intake and digestibility of green forages, we recorded the feeding behaviour and rumination in sheep, in order to determine some factors of variation in this behaviour. These observations were made on 174 samples of green forages belonging to 7 plant species (table $\mathrm{r}$ ). 
The main results obtained were the following :

- The daily time spent eating the green forages ranged between 250 and 400 minutes. This length of time particularly decreased when the stage of maturity of the forages was more advanced, $i . e$. when their voluntary intake and digestibility lowered (tables 2 and 4 ).

- The number of meals per day was much variable (4 to 16 ). It varied from one plant species to another and decreased, in particular, with progressing stage of maturity. This decrease was related to that of the dry matter intake level (tables 2 and 5).

- The unitary eating time ( 3 to $7 \mathrm{mn} / \mathrm{g} / \mathrm{kg} \mathrm{P}^{0.75}$ ) increased very slowly with the stage of maturity of the forages. It was higher for grasses than for legumes (tables 2 and 4 ).

- The daily time spent ruminating (300 to $650 \mathrm{mn}$ ) increased with the stage of maturity of the forages and especially with their crude fiber content (tables 6 and 7 ). This length of time seemed to stabilize around a value ranging between 525 and $575 \mathrm{mn}$ per day according to the plant species or the animal, when the crude fiber content of the plant reached about 30 p. Ioo.

- The unitary ruminating time $\left(4\right.$ to $12 \mathrm{mn} / \mathrm{g} / \mathrm{kg} \mathrm{P}^{0.75}$ ) increased fastly when the digestibility and voluntary intake of the forage decreased (tables 6 and 7 ).

- The daily time spent chewing $(600$ to $950 \mathrm{mn}$ ) was relatively constant for a given plant species at progressive stages of maturity. Therefore, the unitary chewing time was closely and conversely related to the dry matter intake level. This unitary length of time might constitute an index of fibrousness characteristic of roughages as proposed by BALCH (I97r) (tables 6 and 8).

The results are discussed and an attempt made to relate them to the well known factors conditioning the physical regulation of appetite in ruminants, such as digestion rate in the rumen and emptying of the latter (scheme $\mathrm{I}$ ).

We also wondered whether the daily time spent ruminating could be a limiting factor of the intake of forage when the crude fiber content of the latter reached $30 \mathrm{p}$. Ioo of the dry matter.

\section{RÉFÉRENCES BIBLIOGRAPHIQUES}

Balch C. C., I97I. Proposal to use time spent chewing as an index of the extent to which diets for ruminants possess the physical property of fibrourness characteristic of roughages. $B r . J . N u t r ., 26, \mathbf{3}^{83}$.

Balch C. C., Campling R. C., r962. Regulation of voluntary food intake in ruminants. Nutr. Abstr. Rev., 32, 669.

Butterworth M. H., I965. Some aspects of the utilization of tropical forages. I. Green elephant grass at various stages of growth. J. Agri. Sci., 65, 233.

Demarquilly C., I965. Facteurs de variations de la quantité d'herbe verte consommée par le mouton. Revue Fourrages n ${ }^{\circ} 22$

Demarquilly C., Weiss Ph., I970. Tableaux de la valeur alimentaire des fourrages. I. N. R. A., S. E. I., Étude $n^{\circ} 42$.

Dulphy J.-P., I97I. Influence du poids vif et du niveau d'ingestion sur le comportement alimentaire et mérycique du mouton. Ann. Zootech., 20, 477-486.

Dulphy J.-P., I972. Étude de quelques relations, entre le mode de conservation du fourrage ingéré et le comportement alimentaire et mérycique des moutons. Ann. Zootech., 21, 429-44I.

FRENS A.-M., I960. Guest lectures. Suppl. to Medlens bl. Norske Veterinaer. foren, $\mathrm{n}^{\mathrm{o}} 4$.

Gordon J.G., I965. The relationship between rumination and the amount of roughages eaten by sheep. J. Agr. Sci., 64, I 5 I-I 55 .

Jarrige R., Demarquilly C., Dulphy J.-P., I973. The voluntary intake of forages. 5th General Meting of the European Grassland Federation, Uppsala, (Suède).

Jean-Blain C., Borvin R., Bost J., I97I. Digestibilité et rumination : effets de la morphine par voie cérébro-ventriculaire chez le Mouton. Ann. Nutr. Aliment., 25, I 2 I-138.

Journet M., (Résultats non-publiés).

KERBAA A., I969. Étude de quelques facteurs de variation du comportement alimentaire et mérycique des raminants. Thèse Doct. Sci. Clermont-I'errand.

Pearce G. R., I967. Changes in particle size in the reticulorumen of sheep. Atus. J. A gric. Res., 18, II9-I 25.

Pearce G. R., Morr R. J., I964. Rumination in sheep. I. The influence of rumination and grinding upon the passage and digestion of food. Aus. J. Agr. Res., 15, 635 .

Ruckeвusch Y., 1963. Recherches sur la végulation centrale, du comportement alimentaire des ruminants. Thèse Doct. Sci. Lyon. 
Ruckebusch Y., Laplace J. P., I967. Sur le déterminisme de la rumination. Bull. Soc. Scienc. Vet. de Lyon, no I, IO9-I2O.

Welch J. G., Appetite control in sheep by indigestible fibers. J. Anim. Sci., 26, 849 .

WELCH J. G., SMrth A. M., Ig69a. Influence of forage quality on rumination time in sheep. J. Anim. Sci., 28, 8І 3-81 8 .

WELCH J. G., SMith A. M., 1969 $b$. Effect of varying amounts of forage intake on rumination. $J$. Anim. Sci., 28, 827-830. 